ARTICLE

Received 8 Apr 2016 | Accepted 8 Jul 2016 | Published 26 Aug 2016

DOI: $10.1038 /$ ncomms12520

OPEN

\title{
Colloidal cholesteric liquid crystal in spherical confinement
}

Yunfeng Li ${ }^{1}$, Jeffrey Jun-Yan Suen ${ }^{1}$, Elisabeth Prince ${ }^{1}$, Egor M. Larin ${ }^{1}$, Anna Klinkova ${ }^{1}$, Héloïse Thérien-Aubin ${ }^{1}$, Shoujun Zhu², Bai Yang ${ }^{2}$, Amr S. Helmy ${ }^{3}$, Oleg D. Lavrentovich ${ }^{4}$ \& Eugenia Kumacheva ${ }^{1,5,6}$

The organization of nanoparticles in constrained geometries is an area of fundamental and practical importance. Spherical confinement of nanocolloids leads to new modes of packing, self-assembly, phase separation and relaxation of colloidal liquids; however, it remains an unexplored area of research for colloidal liquid crystals. Here we report the organization of cholesteric liquid crystal formed by nanorods in spherical droplets. For cholesteric suspensions of cellulose nanocrystals, with progressive confinement, we observe phase separation into a micrometer-size isotropic droplet core and a cholesteric shell formed by concentric nanocrystal layers. Further confinement results in a transition to a bipolar planar cholesteric morphology. The distribution of polymer, metal, carbon or metal oxide nanoparticles in the droplets is governed by the nanoparticle size and yields cholesteric droplets exhibiting fluorescence, plasmonic properties and magnetic actuation. This work advances our understanding of how the interplay of order, confinement and topological defects affects the morphology of soft matter.

\footnotetext{
${ }^{1}$ Department of Chemistry, University of Toronto, Toronto, Ontario, Canada M5S 3H6. ${ }^{2}$ State Key Laboratory of Supramolecular Structure and Materials, College of Chemistry, Jilin University, Changchun 130012, China. ${ }^{3}$ The Edward S. Rogers Sr. Department of Electrical and Computer Engineering and the Institute of Optical Sciences, University of Toronto, Toronto, Ontario, Canada M5S 3G4. ${ }^{4}$ Liquid Crystal Institute and Chemical Physics Interdisciplinary Program, Kent State University, Kent, Ohio 44242, USA. ${ }^{5}$ Institute of Biomaterials \& Biomedical Engineering, University of Toronto, 164 College Street, Toronto, Ontario, Canada M5S 3G9. ${ }^{6}$ Department of Chemical Engineering and Applied Chemistry, University of Toronto, 200 College Street, Toronto, Ontario, Canada M5S 3E5. Correspondence and requests for materials should be addressed to O.D.L. (email: olavrent@kent.edu) or to E.K. (email: ekumache@chem.utoronto.ca).
} 
P rogrammable organization of nanoparticles (NPs) in new structures is an important target of materials science and nanotechnology ${ }^{1-3}$. The organization of NPs in constrained geometries such as thin films or narrow channels is a valuable strategy for the generation of novel structures with potentially useful properties ${ }^{4-8}$. Spatial constraints can break the symmetry of confined structures and lead to a strong deviation from an equilibrium morphology. Confinement-mediated organization of particles in spherical space, e.g., in spherical droplets is of particular interest, since it offers the conditions of an isotropic confinement and controlled curvature for fundamental studies of particle packing ${ }^{9}$, self-assembly ${ }^{10,11}$ and relaxation of colloidal liquids ${ }^{12}$. Practically, phase separation of colloidal dispersions in spherical confinement can be used for the generation of multicomponent particles, for example, Janus particles ${ }^{13}$. Self-assembly of colloids in droplets paves the way for producing patchy structures mimicking atoms and molecules ${ }^{14}$.

Spherical confinement of liquid crystals formed by NPs offers a large parameter space for the generation of new structures. Macroscopic liquid crystals formed by viruses ${ }^{15,16}$ or carbon nanotubes ${ }^{17,18}$ have been reported; however, the organization of colloidal liquid crystals in spherical droplets remains a largely unexplored area of research, especially, for cholesteric (Ch) phases. Droplets of colloidal Ch liquid crystals can exhibit topological defects that can be used for fundamental studies of soft matter ${ }^{19,20}$ and as templates for the organization of molecules ${ }^{21}$ and $\mathrm{NPs}^{22,23}$. In comparison with molecular mesogens, the organization of NPs in liquid crystalline droplets adds a new set of length scales, thus making these droplets act as hosts for other types of NPs. Moreover, since the interplay of elastic and surface energies in droplets of thermotropic liquid crystals and the coupling of the properties of liquid crystals and NPs may yield structures with new optical and photonic properties ${ }^{24-26}$, these studies can be extended to colloidal lyotropic systems, especially, if their birefringence is enhanced by additives ${ }^{6}$, or by increasing NP volume fraction ${ }^{27}$.
Here we report the organization of rod-shape cellulose nanocrystals (CNCs) in Ch liquid crystals in the spherical confinement of micrometer-size droplets. In marked difference with droplets formed by thermotropic $\mathrm{Ch}$ mesogens ${ }^{28}$, confinement of the $\mathrm{Ch}-\mathrm{CNC}$ liquid crystal results in phase separation into an isotropic phase in the droplet core and a Ch phase with concentric packing of the CNC layers at the droplet periphery. Further progressive confinement causes a transition to a bipolar planar $\mathrm{Ch}$ structure. For the droplets used as hosts for polymer, metal, carbon and metal oxide nanocolloids, we show the capability to control NP partition in the isotropic core and $\mathrm{Ch}$ shell and form $\mathrm{Ch}-\mathrm{CNC}$ droplets with plasmonic, fluorescence and magnetic properties, respectively.

\section{Results}

Spherical droplets of Ch colloidal liquid crystal. Figure 1a shows a transmission electron microscopy (TEM) image of negatively charged, rod-like CNCs with an average length $L$ and diameter $D$ of 183 and $23 \mathrm{~nm}$, respectively. An aqueous $7 \mathrm{wt} \%$ CNC suspension, with a volume fraction of CNCs of 0.046 , was equilibrated for 21 days. Following equilibration, the suspension phase separated into two co-existing phases (Fig. 1b), in agreement with earlier reports ${ }^{29,30}$, that is, into an isotropic top phase with the volume fraction of CNCs of 0.044 and a Ch bottom phase with the volume $\mathrm{CNC}$ fraction $\phi_{0}=0.048$. The volume fraction of the Ch phase in this two-phase system was 0.55 (Supplementary Fig. 1a). In the $\mathrm{Ch}$ phase, the CNCs exhibited long-range orientational order combined with their helical alignment. Polarized optical microscopy (POM) images of the $\mathrm{Ch}$ phase showed a multidomain mosaic pattern with characteristic stripes, with the axis of the Ch helicoid distorted in space (Supplementary Fig. 1b,c). The pitch of the Ch phase measured as a double distance between two adjacent stripes was $P=6.0 \pm 0.3 \mu \mathrm{m}$.

The Ch phase was separated from the two-phase system and emulsified in a microfluidic flow-focusing droplet generator ${ }^{31}$. a

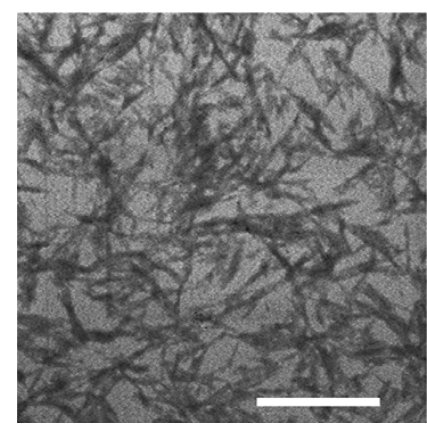

b

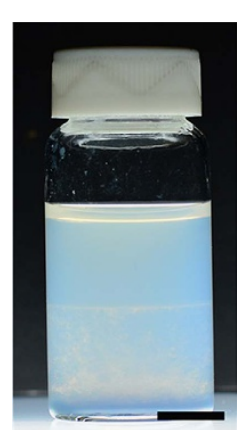

C

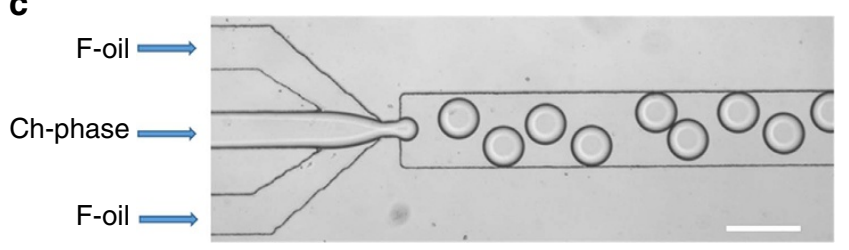

d

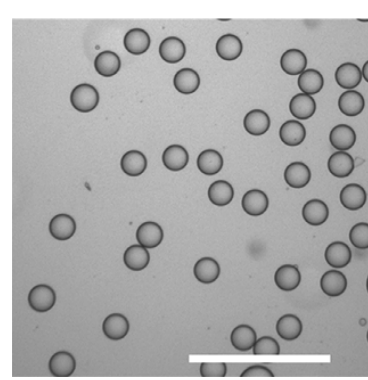

f

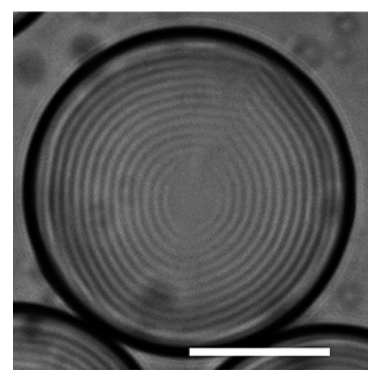

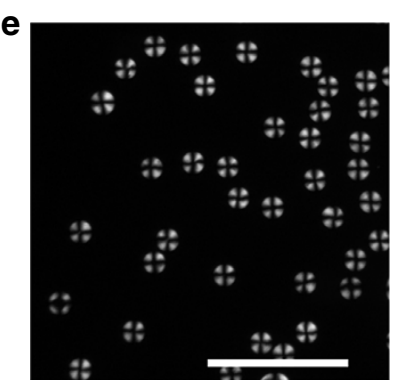

$\mathbf{g}$

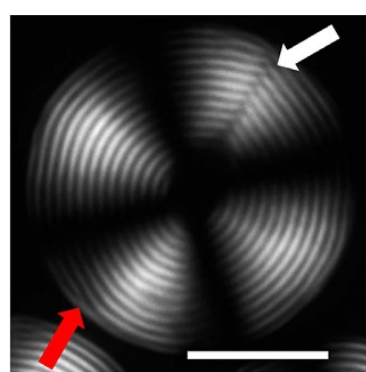

Figure 1 | Generation of droplets by microfluidic emulsification of the Ch-CNC suspension. (a) TEM images of dried CNCs. The scale bar is $500 \mathrm{~nm}$. (b) Phase separation of the macroscopic CNC suspension into a Ch phase (bottom) and an isotropic phase (top). The scale bar is $1 \mathrm{~cm}$. (c) Optical microscopy image of microfluidic generation of droplets of the Ch-CNC phase. The flow rate of F-oil and the $\mathrm{Ch}-\mathrm{CNC}$ phase were 0.8 and $0.2 \mathrm{ml} \mathrm{h}^{-1}$ respectively. The scale bar is $250 \mu \mathrm{m}$. (d,f) Bright-field (BF) optical microscopy images and (e,g) corresponding polarized optical microscopy (POM) images of Ch-CNC droplets with $\phi_{0}=0.048$. Scale bars are $500 \mu \mathrm{m}(\mathbf{d}, \mathbf{e})$ and $50 \mu \mathrm{m}(\mathbf{f}, \mathbf{g})$. Arrows in $\mathbf{g}$ show the radial disclination line defect running perpendicularly to the Ch layers (white arrow) and an edge dislocation running parallel to the Ch layers (red arrow). 
A fluorinated oil (F-oil) containing $1.0 \mathrm{wt} \%$ of a surfactant triblock copolymer perfluoropolyether and poly(ethylene oxide-co-propylene oxide) and the $\mathrm{Ch}$ phase were supplied to the microfluidic device as a continuous and droplet phases, respectively (Fig. 1c). In the orifice, the shear stress imposed by the two outer F-oil streams on the inner thread of the Ch phase resulted in a periodic breakup of the aqueous stream into uniformly sized droplets with a polydispersity of $2.5 \%$. The droplet radius, $R$, was varied from 40 to $60 \mu \mathrm{m}$ by changing the volumetric flow rate of F-oil from 1.5 to $0.3 \mathrm{mlh}^{-1}$ at a constant flow rate of the $\mathrm{Ch}$ phase at $0.2 \mathrm{ml} \mathrm{h}^{-1}$.

Figure 1d-g shows the bright-field $(\mathrm{BF})$ and the POM images of the droplets with a radius $R=45 \mu \mathrm{m}$. The POM images showed the characteristic features of the $\mathrm{Ch}$ phase, that is, a Maltese cross and a shell with alternating bright and dark concentric rings corresponding to the surfaces of constant refractive index ${ }^{26}$ (a similar ring pattern is shown in the BF image in Fig. 1f). This structure of the droplet shell corresponded to the tangential $\mathrm{CNC}$ alignment at the droplet/F-oil interface with a radial orientation of the helical axis $\hat{\mathbf{h}}$ showing the direction along which the local orientation of the CNCs twists in space. The local orientation of the CNCs was described by the so-called director field $\hat{\mathbf{n}}$. (Note that $\hat{\mathbf{n}}$ and $\hat{\mathbf{h}}$ were orthogonal to each other). The average pitch $P$ in the $\mathrm{Ch}-\mathrm{CNC}$ droplets, measured as a double distance between two adjacent concentric rings, was $6.1 \pm 0.3 \mu \mathrm{m}$, that is, close to $P=6.0 \pm 0.3 \mu \mathrm{m}$ of the macroscopic Ch phase. The central region of the droplets with a radius of $8.5 \pm 1.1 \mu \mathrm{m}$ was formed by the isotropic phase. The equilibrium droplet structure was reached via transient $\operatorname{states}^{32}$. Spherical concentric packing of the Ch layers started at the droplet/F-oil interface, being assisted by the tangential surface anchoring of the CNCs, and with equilibration time, it gradually propagated toward the droplet centre (Supplementary Fig. 2).

Usually, confinement of rods between parallel planar substrates is expected to enhance the orientational order of the system ${ }^{33,34}$; however, spherical confinement of $\mathrm{Ch}$ phases leads to a different situation, in which the equilibrium state must be accompanied by a particular minimum number of defects, as dictated by topology. A Ch droplet with an infinitely strong tangential anchoring of the director at the droplet surface should acquire spherical packing of Ch pseudo-layers, which implies that the normal $\hat{\mathbf{h}}$ to these layers forms a radial point-defect hedgehog with the core in the droplet centre ${ }^{32}$. In such droplets, the director within each spherical pseudo-layer must contain defects, according to the Euler-Poincare theorem ${ }^{32}$. When the spherical pseudo-layers are confined in a concentric manner within the Ch droplet, these point defects form radial defects-disclinations-in the local director field $\hat{\mathbf{n}}$ (ref. 32). Furthermore, if the equilibrium is not perfect, the Ch droplet may contain additional defects, such as dislocations that are associated with, for example, partial insertion or removal of the concentric layers.

Thus the equilibrium state of the $\mathrm{Ch}-\mathrm{CNC}$ droplets with a tangential alignment of the director at the $\mathrm{Ch} / \mathrm{F}$-oil interface could not be realised without a point defect in the radial distribution of the normal $\hat{\mathbf{h}}$, accompanied by the radial disclination lines in the director field $\hat{\mathbf{n}}$. Near the equilibrium, dislocation defects were also possible.

The experimental results, in general, supported these expectations. In Fig. 1f,g, the point defect is evidenced by the concentric packing of the $\mathrm{Ch}-\mathrm{CNC}$ pseudo-layers (which implies a radial configuration of the field $\hat{\mathbf{h}}$ ). The equilibrium disclination is marked with a white arrow, while an occasionally encountered dislocation is marked with a red arrow. The equilibrium disclinations were necessitated by the spherical confinement topology in the regime of strong chirality, when $R>>P^{35,36}$. As demonstrated in the next section, this ideal structure expected on the basis of topological considerations was realised only partially, since the structure of the $\mathrm{Ch}-\mathrm{CNC}$ droplets exhibited a strong dependence on their size.

Size dependence of the internal structure of $\mathrm{Ch}-\mathrm{CNC}$ droplets. The effect of confinement on the droplet morphology was examined as a function of the droplet size for three cases.

Very-large drops with $R>115 \mu$ m exhibited a multidomain $\mathrm{Ch}$ structure, similar to the macroscopic $\mathrm{Ch}-\mathrm{CNC}$ phase (Supplementary Fig. 3). A multidomain morphology of large droplets with the axis of the $\mathrm{Ch}$ helicoid distorted in space was a manifestation of a very-long time needed for their complete equilibration. In this regard, the very-large droplets behaved similarly to the macroscopic $\mathrm{Ch}-\mathrm{CNC}$ phase.

Inspection of droplets with a radius in the range $5 \leq R \leq 115$ $\mu \mathrm{m}$ revealed that they had three types of 'monodomain' structures $($ Fig. 2a-c). The corresponding schematics of the CNC orientation in these droplets are shown in Fig. 2d-f, where the Ch layers are presented as surfaces separated by half-pitch. The distribution of the populations of droplets with different morphologies is shown in Fig. $2 \mathrm{~g}$.

For large droplets with dimensions in the range $40 \leq R \leq 115$ $\mu \mathrm{m}(6.5 \leq R / P \leq 18.8), \sim 96 \%$ of the droplets exhibited a Ch shell with a concentric packing of CNC pseudo-layers and thus radial configuration of the normal $\hat{\mathbf{h}}$ that formed a radial point-defect hedgehog. In these droplets, we observed either one radial disclination of strength 2 (Fig. $1 \mathrm{~g}$ ) or two radial disclinations of strength 1 each (Supplementary Fig. 4). In both cases, the total topological charge was two, equal to the Euler characteristic of the sphere, as required by the Euler-Poincare theorem ${ }^{32}$. A single radial disclination was observed in $\sim 90 \%$ of the droplets, in agreement with numerical simulations and experimental observations of $\mathrm{Ch}$ droplets formed by thermotropic molecular liquid crystals ${ }^{28,37}$.

The most striking feature of the droplets with a concentric $\mathrm{Ch}$ layer packing was that the radial point defect exhibited an isotropic core with a radius, $r_{\mathrm{i}}$, in the range from $\sim 3$ to $8.3 \mu \mathrm{m}$ ( 0.5-1.4 P; Fig. 2a,d). The size of the core was larger in smaller droplets (Supplementary Fig. 5). With the droplet radius $R$ decreasing from 115 to $40 \mu \mathrm{m}$, the volume fraction $\sim r_{i}^{3} / R^{3}$ of the isotropic core in the droplet increased from $2 \times 10^{-5}$ to $9 \times 10^{-3}$ (Fig. 3a). This result suggested that spherical confinement caused phase separation in the system that exhibited a single-phase $\mathrm{Ch}$ behaviour in macroscopic samples, with the isotropic phase appearing at the core of the radial point defect. It was thus of interest to explore whether the same spherical confinement conditions would cause a change in the $\mathrm{Ch}$ pitch in the droplets. Fig. $3 \mathrm{~b}$ shows the effect of the local curvature $1 / R_{\mathrm{loc}}$ on the pitch of the Ch shell, where $R_{\text {loc }}$ was measured from POM images as the radial distance between the droplet centre and bright concentric rings in the $\mathrm{Ch}$ shell, starting from the second ring. For droplets with a radius of 45 and $100 \mu \mathrm{m}$, filled with the Ch phase at $\phi_{0}=0.048$, with the local curvature increasing from 0.01 to 0.08 , the pitch remained invariant at $P \approx 6.1 \mu \mathrm{m}$. On the other hand, similarly to the macroscopic Ch-CNC phase ${ }^{29}$, the $\mathrm{Ch}$ pitch in the droplets depended on the $\mathrm{CNC}$ concentration: when $\phi_{0}$ increased from 0.036 to 0.054 , the pitch changed from 7.9 to $5.2 \mu \mathrm{m}$ (Fig. 3c).

Intermediate-size droplets with $10 \leq R \leq 40 \mu \mathrm{m}$ exhibited a transitional 'ellipsoidal concentric layer' pattern corresponding to planar $\mathrm{Ch}$ pseudo-layers in the centre and tangential CNC orientation at the droplet periphery (Fig. 2b,e). A large fraction of droplets with $R \leq 20 \mu \mathrm{m} \quad(R / P \leq 3.3)$ displayed a stripe pattern characteristic of flattened $\mathrm{Ch}$ layers trapped between the two diametrically opposite poles (Fig. 2c). Notably, the 

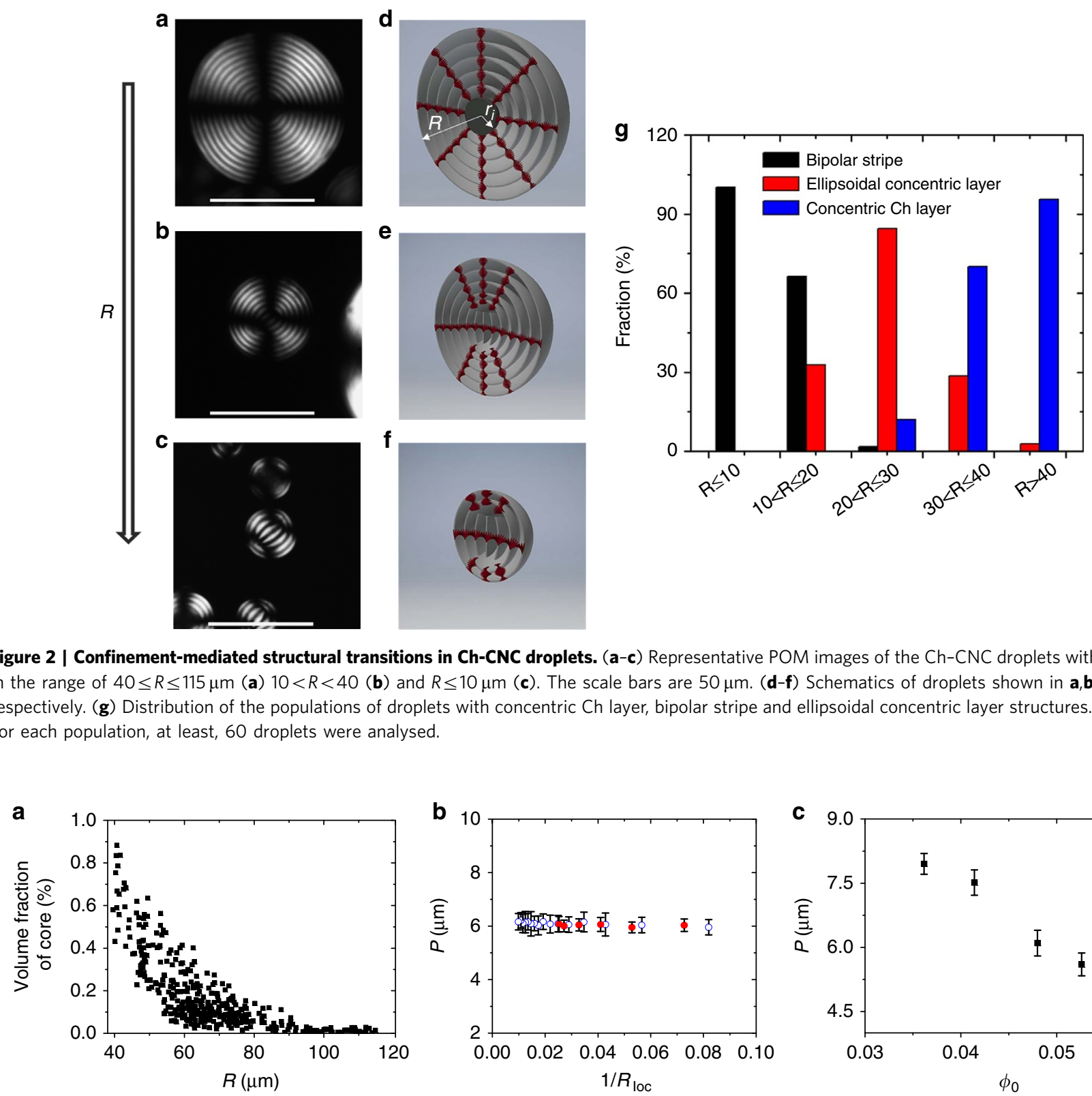

Figure 2 | Confinement-mediated structural transitions in Ch-CNC droplets. (a-c) Representative POM images of the Ch-CNC droplets with dimensions in the range of $40 \leq R \leq 115 \mu \mathrm{m}$ (a) $10<R<40$ (b) and $R \leq 10 \mu \mathrm{m}$ (c). The scale bars are $50 \mu \mathrm{m}$. (d-f) Schematics of droplets shown in a,b and $\mathbf{c}$, respectively. (g) Distribution of the populations of droplets with concentric $\mathrm{Ch}$ layer, bipolar stripe and ellipsoidal concentric layer structures. $\phi_{0}=0.048$. For each population, at least, 60 droplets were analysed.
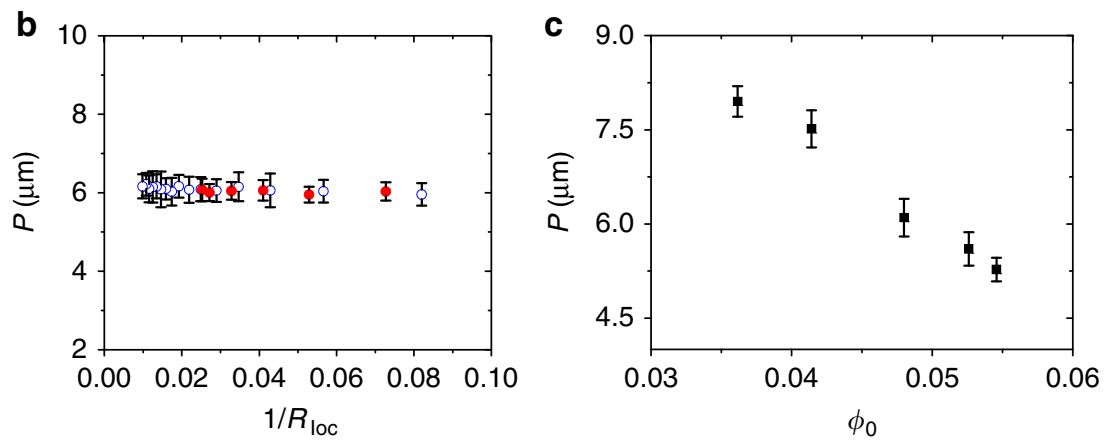

Figure 3 | Structural characteristics of droplets with concentric Ch layers. (a) Variation in the volume fraction of the isotropic core, plotted as a function of droplet radius $R$, based on the analysis of 390 droplets. (b) Pitch variation in the Ch-CNC droplets with a radius of 45 (red spheres) and $100 \mu \mathrm{m}$ (blue circles), plotted as a function of the local curvature $\left(1 / R_{\mathrm{loc}}\right)$ of the droplet. $R_{\mathrm{loc}}$ was measured from POM images as the radial distance between the droplet centre and bright even rings in the Ch shell, starting from the second one. The error bars represent the s.d. For each population of droplets, at least, 10 droplets were analysed. In $\mathbf{a}, \mathbf{b} \phi_{0}=0.048$. (c) Pitch variation plotted as a function of CNC volume fraction in the droplets. For each experimental point, at least, 20 droplets were analysed. The error bars represent the s.d.

interpretation of POM images for the intermediate-size droplets and for the small droplets with $R \leq 10 \mu \mathrm{m}$ (see below) depended on droplet orientation: when the axis of the Ch structure pointed up, a concentric ring structure was observed. Thus the morphology of these droplets was examined by carefully rotating them in the fluid cell (see, for example, Supplementary Movie 1).

Small droplets with $R \leq 10 \mu \mathrm{m}$ exhibited almost exclusively a bipolar stripe pattern. For such droplets, at $\phi_{0}=0.048$, the pitch remained invariant at $7.1 \mu \mathrm{m}$ with increasing droplet size (Fig. 4a). When the droplets were formed from the $\mathrm{Ch}-\mathrm{CNC}$ phase with varying $\phi_{0}$, the pitch showed the dependence on the volume fraction of the CNCs: an increase in $\phi_{0}$ from 0.036 to
0.054 resulted in the reduction of the pitch from 9.1 to $5.7 \mu \mathrm{m}$, respectively (Fig. $4 \mathrm{~b}$ ).

The transition from the ellipsoidal concentric layers to flattened Ch layers occurred at approximately $R_{\mathrm{tr}} \approx 10 \mu \mathrm{m}$. For the droplet size of $R_{\mathrm{tr}}$, the elastic energy of spherical packing of the Ch-CNC layers that scales as $K R$, and the surface anchoring energy of the planar CNC layers that scales as $W R^{2}$, were approximately equal to each other ${ }^{32}$. The balance of these two energies enabled the estimation of the surface anchoring strength, $W$, at the Ch droplet/F-oil interface, as $W \approx K / R_{t r} \approx 10^{-6} \mathrm{Jm}^{-2}$, where $K$ is the average value of the Frank elastic constant that was assumed to be on the order of $10 \mathrm{pN}$ (refs 38,39), as for other lyotropic liquid crystals. 

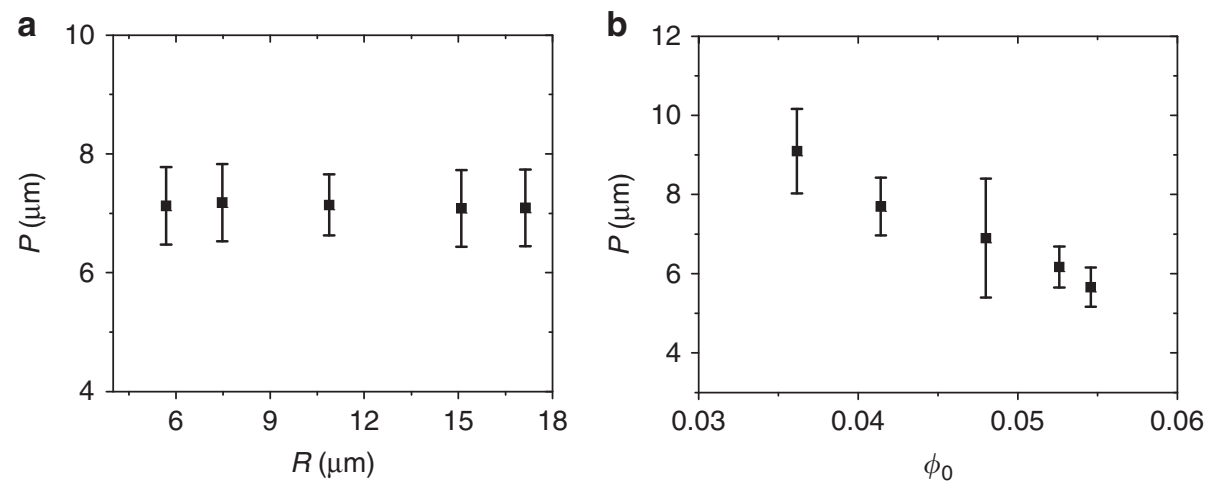

Figure 4 | Structural characteristics of the droplets with bipolar patterns. (a) Variation in pitch, plotted as a function of droplet radius $R . \phi_{0}=0.048$. (b) Pitch variation as a function of CNC volume fraction in the droplets. For each experimental point, at least, 30 droplets were analysed. The error bars represent the s.d.

Distribution of nanocolloids in the Ch-CNC droplets. In assumption that the micrometer-size isotropic core in the $\mathrm{Ch}-\mathrm{CNC}$ droplets can be used for the incorporation of chemicals or other nanocolloids, we loaded the droplets with spherical negatively charged 54 and $184 \mathrm{~nm}$-diameter fluorescent dye-labelled polystyrene latex NPs and examined the effect of NP size on their distribution within the droplets. In equilibrium, a macroscopic mixture of the Ch-CNC phase and latex NPs phase separated into a latex-rich isotropic phase and a CNC-rich Ch phase (Supplementary Fig. 6 and Supplementary Note 1). The volume fraction of the isotropic phase increased at a higher volume fraction of latex NPs, $\phi_{\mathrm{NP}}$.

Droplets were generated in the microfluidic droplet generator immediately after mixing the $\mathrm{Ch}$ phase and latex NPs at $\phi_{\mathrm{NP}}=0.0052$ and subsequently, equilibrating droplets for $72 \mathrm{~h}$. In the droplets, the NPs segregated in the cores of disclinations, similarly to the effect described for disclinations in thermotropic systems $^{21,40,41}$ and in the isotropic core of the radial hedgehog (Supplementary Figs 7 and 8, respectively). The Ch shell structure of the droplets with a pitch $P$ of $\sim 7 \mu \mathrm{m}$ was preserved. The extent of partition in the droplet core increased with NP dimensions (Fig. 5a-h and Supplementary Fig. 9a-d). Examination of the variation of the ratio of the core-to-shell fluorescence intensities, based on the fluorescence microscopy (FM) images of the droplets revealed that $\sim 70$ and $\sim 93 \%$ of 54 and $184 \mathrm{~nm} \mathrm{NPs}$, respectively, resided in the isotropic core of the hedgehog defect (Fig. 5j,k). Because of the stronger phase separation between the CNCs and the latex NPs with increasing latex content in the system, the extent of latex segregation in the isotropic core increased at higher $\phi_{\mathrm{NP}}$ (Fig. $5 \mathrm{k}$ and Supplementary Figs 10-12), and the radius of the isotropic core increased from 12 to $30 \mu \mathrm{m}$ (Fig. $5 \mathrm{j}$ and Supplementary Fig. 11). In contrast with small latex NPs, larger microspheres with a diameter of $1,000 \mathrm{~nm}$ formed multi-particle aggregates randomly distributed within the droplets (Fig. 6 and Supplementary Fig. 9e,f).

With the goal to extend the range of functionalities of the $\mathrm{Ch}-\mathrm{CNC}$ droplets, we examined the organization of plasmonic gold NPs, fluorescent carbon NPs (carbon dots) and magnetic iron oxide rod-shape NPs, in the $\mathrm{Ch}-\mathrm{CNC}$ droplets. In comparison with molecular $\mathrm{Ch}$ liquid crystals, a relatively large ( $\sim 30 \mathrm{~nm}$ (ref. 29)) average spacing between the CNCs in the $\mathrm{Ch}-\mathrm{CNC}$ phase could accommodate small-size NPs. Droplets loaded with NPs were generated by the microfluidic emulsification of the $\mathrm{Ch}$ phase mixed with NPs. Figure 7a, top and bottom, shows transmission electron microscopy images of 10 and $50 \mathrm{~nm}$ gold NPs capped with thiol- terminated poly(ethylene glycol) with a molecular weight $M_{\mathrm{n}}=5,000 \mathrm{~g} \mathrm{~mol}^{-1}$. Small $10 \mathrm{~nm}$ gold NPs exhibited a uniform spatial distribution (Fig. 7b, top) in the core-shell Ch-CNC droplets, while $50 \mathrm{~nm}$ NPs partitioned in the isotropic droplet centre (Fig. 7b, bottom). Extinction spectra of the droplets loaded with gold NPs showed surface plasmon resonance a wavelengths similar to the corresponding bands of NPs in a macroscopic aqueous dispersion (Fig. 7c), indicating good NP dispersibility in the droplets and the capability to form plasmonic Ch-CNC droplets, with the NPs residing either in the Ch phase, or in the isotropic phase.

Droplets loaded with $\sim 3.5 \mathrm{~nm}$-size fluorescent carbon dots (Fig. 7d) exhibited a uniform distribution in the Ch-CNC droplets, based on the distribution of fluorescence intensity within the droplets (Fig. 7e and Supplementary Fig. 13). The droplets exhibited a green emission colour when the NPs were excited at $\lambda_{\text {exc }}=440 \mathrm{~nm}$ (Fig. $7 \mathrm{f}$ ), while blue and red emission colours were obtained at $\lambda_{\text {exc }}$ of 365 and $550 \mathrm{~nm}$, respectively (Supplementary Fig. 14), due to the intrinsic excitation-dependent properties of carbon dots ${ }^{42}$.

In contrast with small gold NPs and carbon dots, magnetic $\mathrm{Fe}_{3} \mathrm{O}_{4} / \mathrm{SiO}_{2}$ rods with an average diameter and length of $100 \mathrm{~nm}$ and $1 \mu \mathrm{m}$, respectively (Fig. $8 \mathrm{a}$ ), formed micrometer-size aggregates in the droplets (shown in Fig. 8b, top). This effect was consistent with the aggregation of large $1,000 \mathrm{~nm}$ latex microspheres in the $\mathrm{Ch}-\mathrm{CNC}$ droplets. These aggregates moved towards a magnet placed outside the cell filled with the emulsion of the $\mathrm{Ch}-\mathrm{CNC}$ droplets loaded with magnetic rods (Fig. 8b, bottom). Both translational motion of the droplets with acceleration of $1.7 \mu \mathrm{ms}^{-2}$ (Fig. $8 \mathrm{c}$ and Supplementary Movie 2) and rotational droplet motion (Supplementary Movie 3) were achieved under the action of magnetic field.

\section{Discussion}

The existence of a large isotropic spherical region in the $\mathrm{Ch}$ droplet centre (Fig. 2a) was in striking difference with enhanced order in nematic phases confined between planar surfaces ${ }^{33,34}$. We ascribe this difference to the spherical confinement of the $\mathrm{Ch}-\mathrm{CNC}$ phase, which in equilibrium resulted in defects such as linear disclinations and the radial point-defect hedgehog. In contrast with a spherical confinement, an ideal flat confinement in equilibrium would not require any defects, nor isotropic inclusions, since the Ch phase separated at the binodal from its isotropic counterpart should reach a homogeneous structure at equilibrium.

Confinement by the spherical $\mathrm{Ch} / \mathrm{F}$-oil interface imposed spherical curvature of the $\mathrm{Ch}$ pseudo-layers, due to the finite 

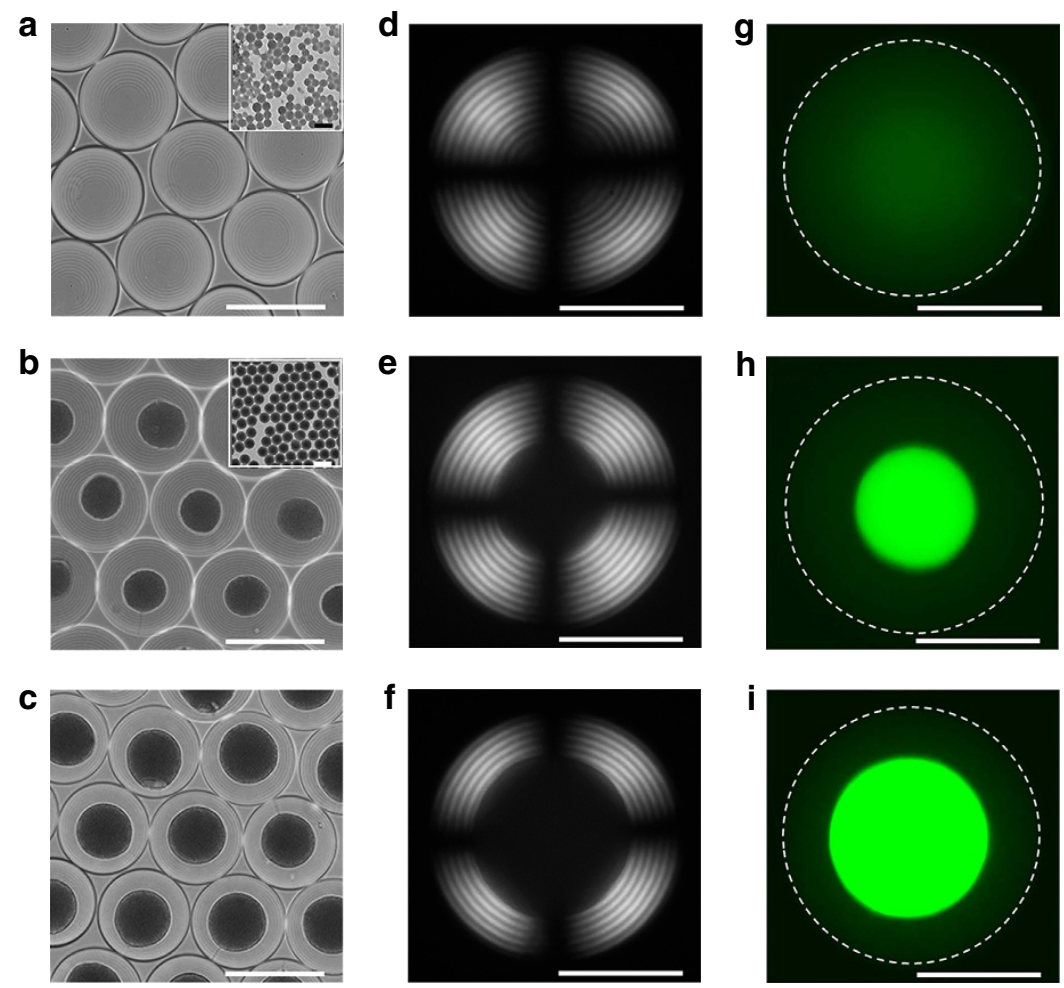

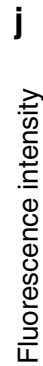

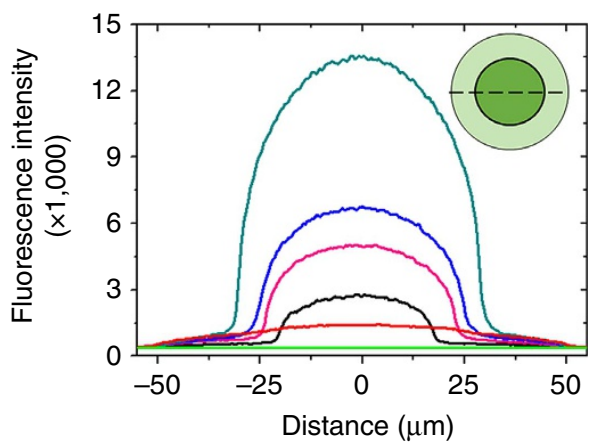

$\mathbf{K}$

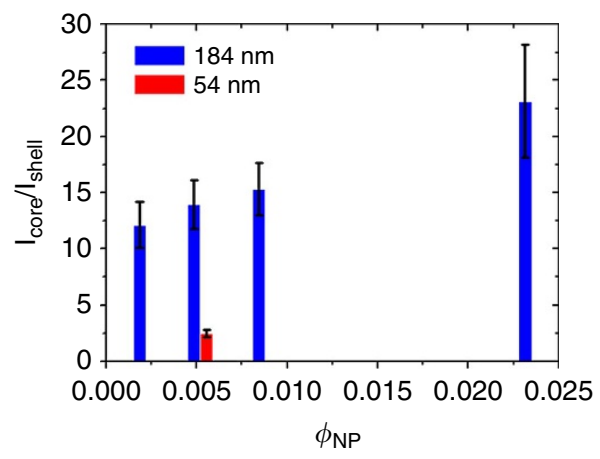

Figure 5 | Loading of Ch-CNC droplets with latex NPs. BF (a-c), POM (d-f) and FM ( $\mathbf{g}-\mathbf{i})$ images of droplets containing fluorescein isothiocyanate dyelabelled $54 \mathrm{~nm}$ latex NPs at $\phi_{\mathrm{NP}}=0.0052(\mathbf{a}, \mathbf{d}, \mathbf{g})$ and $184 \mathrm{~nm}$ dye-labelled latex NPs at $\phi_{\mathrm{NP}}$ of $0.0052(\mathbf{b}, \mathbf{e}, \mathbf{h})$ and 0.0235 (c,f,i). The scale bars in $\mathbf{a}-\mathbf{c}$ are $100 \mu \mathrm{m}$; the scale bars in $\mathbf{d}-\mathbf{i}$ are $50 \mu \mathrm{m}$. Insets in $\mathbf{a}, \mathbf{b}$ show the corresponding TEM images of 54 and $184 \mathrm{~nm}$ latex NPs. The scale bar in insets is $100 \mathrm{~nm}$ in $\mathrm{a}$ and $300 \mathrm{~nm}$ in b. (j) Representative fluorescence line profiles of the Ch-CNC droplets loaded with $54 \mathrm{~nm}$ latex NPs at $\phi_{\mathrm{NP}}=0.0052$ (red) and $184 \mathrm{~nm}$ NPs at $\phi_{\mathrm{NP}}$ of 0 (green), 0.0022 (black), 0.0052 (magenta), 0.0088 (blue) and 0.0235 (dark cyan). Inset shows the schematic of a droplet with a fluorescence line profile drawn through the droplet centre (dashed line). To prepare droplets with $\phi_{\mathrm{NP}}=0$, the Ch-CNC phase was mixed with a supernatant of the latex dispersion. (k) Variation in the core-to-shell fluorescence intensity ratio plotted as a function of NP volume fraction in the droplets. In $\mathbf{a}-\mathbf{k}, \phi_{0}=0.043$. For each value of $\phi_{\mathrm{NP}}$ at least, 30 droplets were analysed. The error bars represent the s.d.

surface anchoring that sets the helical axis $\hat{\mathbf{h}}$ perpendicular to the interface. To realign $\hat{\mathbf{h}}$ into a tangential orientation, one would need to perform the work against the anchoring energy that scales as $W R^{2}$ (ref. 32). On the other hand, the energy of elastic distortions associated with the spherical curvature of pseudo-layers within the droplet scales as $K R$. Therefore, droplets with a large radius, $R>K / W$, would tend to preserve concentric spherical packing of the Ch pseudo-layers, while satisfying the boundary anchoring conditions, with $\hat{\mathbf{h}}$ being orthogonal to the $\mathrm{Ch} / \mathrm{F}$-oil interface. For $R>K / W$, the anchoring energy penalty is significantly larger than the elastic cost of the concentric packing, that is, $W R^{2}>>K R$. Thus for the surface anchoring energy $W=10^{-6} \mathrm{Jm}^{-2}$ and the elastic constant $K=10 \mathrm{pN}$, droplets with a radius $R$ larger than $R_{\mathrm{tr}}=K / W \sim 10 \mu \mathrm{m}$ tend to curve the Ch layers and form concentric spherical layers, to preserve the surface anchoring conditions. On the other hand, when $R<K / W$, the structure with flattened layers is energetically preferred, that is, $W R^{2}<<K R$.

The spherical packing of the $\mathrm{Ch}$ layers implies a radial configuration of the normal $\hat{\mathbf{h}}$ to the $\mathrm{Ch}$ layers, with the components $\hat{\mathbf{h}}=(x, y, z) / \sqrt{x^{2}+y^{2}+z^{2}}$ written in the Cartesian coordinates. As one approaches the centre of the droplet at $(x, y, z)=(0,0,0)$, the spatial gradients of $\hat{\mathbf{h}}$ and thus the elastic energy density strongly increase. Such an increase can be avoided if the regions with strong gradients adopt a less ordered structure, by e.g., local melting into an isotropic phase ${ }^{32}$. The effect of isotropic core has been considered in details theoretically for dislinations ${ }^{43}$ and for radial point defects hedgehogs ${ }^{44}$ formed in spherical volumes of a thermotropic nematic liquid crystal doped with a non-mesogenic additive. Analytical solutions are difficult, but the numerical simulations show that the isotropic core of the point defect can have a radius between 2 and $50 \mathrm{~nm}$ in pentylcyanobiphenyl (5CB), a typical thermotropic nematic liquid crystal $^{44}$. Recent observations of defect loops with a $10 \mathrm{~nm}$ radius formed at the core of point defects in thermotropic liquid crystals support these predictions ${ }^{45}$. In our experiments, we attribute the appearance of a significantly larger core with a radius $r_{i}>1 \mu \mathrm{m}$, to the lyotropic nature of the liquid crystal system with the low concentration of the solute. Interestingly, large defect cores have been observed in another type of lyotropic liquid crystals, the so-called chromonics ${ }^{46}$.

To understand the mechanism of the appearance of the large isotropic core in the lyotropic $\mathrm{Ch}-\mathrm{CNC}$ droplets with confinement-imposed spherical packing, we consider the balance of the elastic, condensation and surface energies associated with the cores, following the earlier models ${ }^{43,44,46,47}$. The free energy of the formation of an isotropic core with a radius $r_{\mathrm{i}}$ in the centre of the Ch droplet with a radius $R>>P$ and concentric packing of the Ch layers in the droplet shell is

$$
F=8 \pi\left(K_{1}-K_{24}\right)\left(R-r_{i}\right)+4 \pi \sigma_{\mathrm{chi}} r_{i}^{2}+4 \pi\left(f_{i}-f_{\mathrm{ch}}\right) r_{i}^{3} / 3,
$$

where the first, the second and the third terms are the energy of elastic distortion of the $\mathrm{Ch}$ layers in the droplet shell, the surface energy of the Ch-isotropic interface, and the free energy difference between the isotropic and $\mathrm{Ch}$ phases, respectively. Here $K_{1}$ and $K_{24}$ are the splay and saddle-splay elastic constants of the Ch phase, 
a

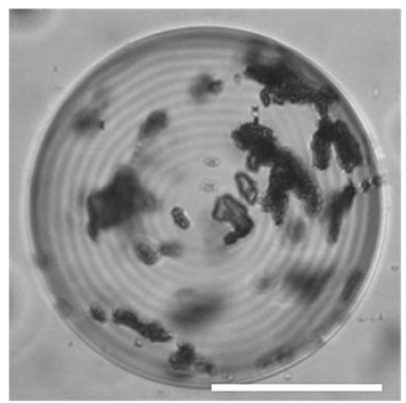

C

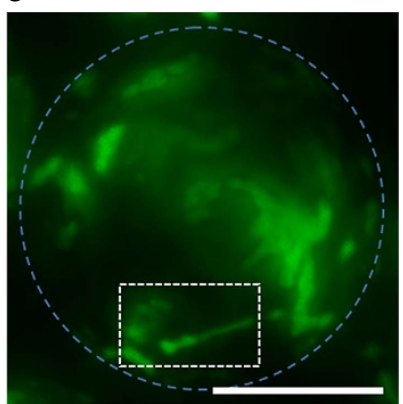

b

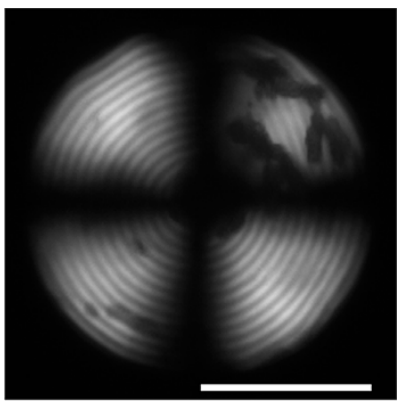

d

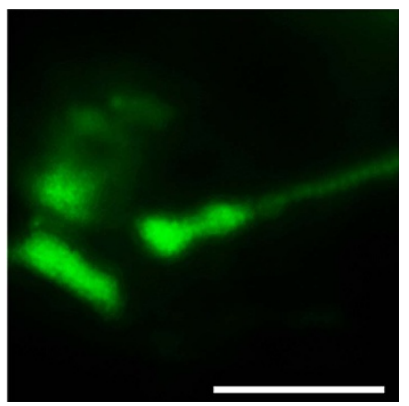

Figure 6 | Ch-CNC droplets loaded with $1000 \mathrm{~nm}$ latex particles. (a) BF, (b) POM and (c) FM images of aggregates of 1,000 nm latex particles in the Ch-CNC droplets with $\phi_{0}=0.043$. $\phi_{\mathrm{NP}}=0.0052$. (d) Enlarged FM image of the latex particle aggregates shown in the white box in $\mathbf{c}$. The scale bar is $50 \mu \mathrm{m}$ in a-c and $15 \mu \mathrm{m}$ in $\mathbf{d}$.

respectively, $\sigma_{\text {chi }}$ is the interfacial tension at the Ch-isotropic interface, and $f_{i}$ and $f_{\text {ch }}$ are the free energy densities of the isotropic and $\mathrm{Ch}$ phases, respectively. In the coarse-grained model ${ }^{32}$ for the gradients of the field $\hat{\mathbf{h}}$ the (volume) free energy density is written as $f_{\text {el }}=\frac{1}{2} K_{1}(\operatorname{div} \hat{\mathbf{h}})^{2}-K_{24} \operatorname{div}(\hat{\mathbf{h}} \operatorname{div} \hat{\mathbf{h}}+\hat{\mathbf{h}} \times \operatorname{curl} \hat{\mathbf{h}})$.

The first term in equation (1) is obtained by integrating the elastic energy density, $f_{\text {el }}$ given above, with $\hat{\mathbf{h}}=\hat{\mathbf{r}}$ and $\operatorname{div} \hat{\mathbf{h}}=2 / r$, over the Ch-CNC shell volume; here $\hat{\mathbf{r}}$ is the radius-vector of the spherical coordinate system, and $r$ is the distance from the droplet centre $^{32}$. Since $K_{1}-K_{24}>0$ (to ensure the flat configuration of the Ch layers of an unbounded system $)^{32}, \sigma_{\text {chi }}>0$ and $f_{\mathrm{i}}-f_{\text {ch }}>0$, the term that favors the existence of the isotropic core is $-8 \pi$ $\left(K_{1}-K_{24}\right) r_{i}$, by which the elastic energy is reduced when the highly curved $\mathrm{Ch}$ layers in the droplet centre are replaced with an isotropic spherical region. High elastic energy of distortions at the droplet centre is the main mechanism leading to the appearance of the isotropic core.

For a lyotropic system, an isotropic core implies that a fraction of CNCs is transferred from the core into the Ch shell. Therefore, equation (1) should be supplemented by the mass conservation $\mathrm{law}^{44}$

$$
\phi_{0} R^{3}=\phi_{i} r_{i}^{3}+\phi_{\mathrm{ch}}\left(R^{3}-r_{i}^{3}\right),
$$

where $\phi_{i}$ and $\phi_{\mathrm{ch}}$ are the CNC volume fractions in the isotropic and $\mathrm{Ch}$ regions of the droplet. For the studied range of radii of the $\mathrm{Ch}-\mathrm{CNC}$ droplets, the volume fraction $\phi_{\mathrm{ch}}$ of the CNC in the $\mathrm{Ch}$ shell does not significantly differ from the CNC volume fraction $\phi_{0}$ of the emulsified single-phase macroscopic Ch-CNC phase. For example, even if all the CNCs were expelled from the isotropic core, for $\left(r_{i} / R\right)^{3}$ as large as $9 \times 10^{-3}$ (which is the case

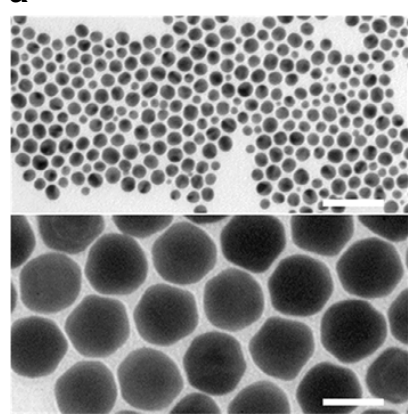

d

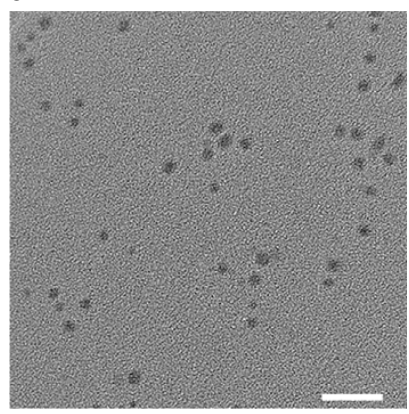

b

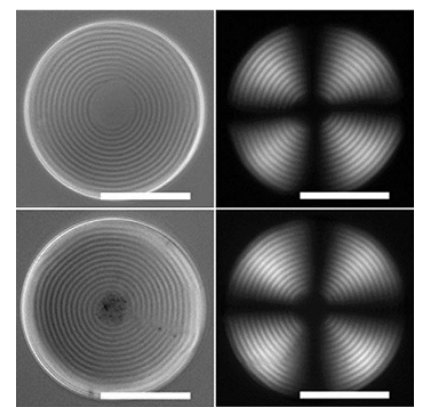

e

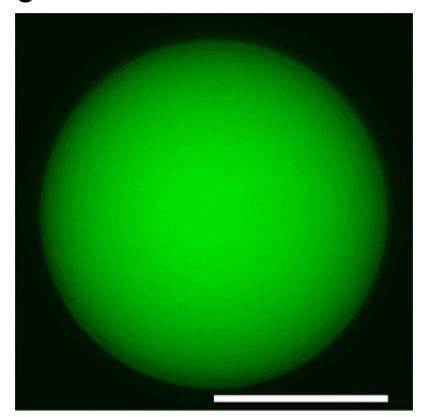

C

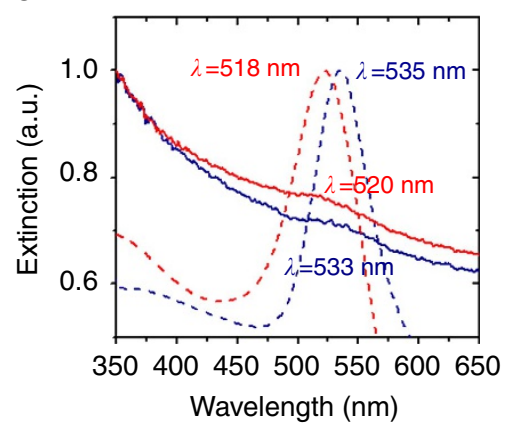

f

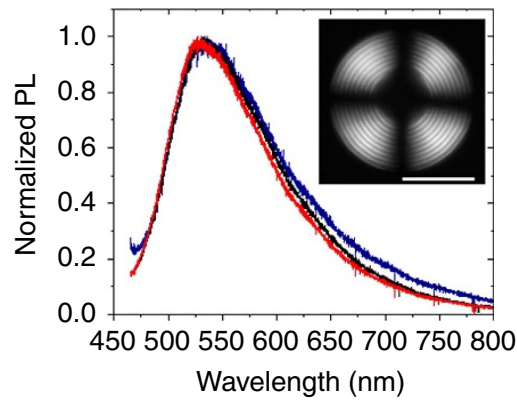

Figure 7 | Co-assembly of CNCs and inorganic nanoparticles. (a) TEM images of $10 \mathrm{~nm}$ (top) and $50 \mathrm{~nm}$ (bottom) gold NPs. The scale bar is $50 \mathrm{~nm}$. (b) BF (left panel) and POM (right panel) images of the Ch-CNC droplets loaded with $10 \mathrm{~nm}$ gold NPs (top panel) and $50 \mathrm{~nm}$ gold NPs (bottom panel). The concentration of gold NPs in the droplets is $0.1 \mathrm{mg} \mathrm{ml}^{-1}$. The scale bar is $50 \mu \mathrm{m}$. (c) Extinction spectra of aqueous gold NP dispersion (dashed lines) and Ch-CNC droplets (solid lines) loaded with $10 \mathrm{~nm}$ (red lines) and $50 \mathrm{~nm}$ (blue lines) gold NPs. The broadening of extinction spectra of the NP-loaded droplets was caused by the light scattering by droplets in the visible light region. (d) TEM images of carbon dots. The scale bar is $20 \mathrm{~nm}$. (e) FM image of the Ch-CNC droplet carrying carbon dots at concentration of $1 \mathrm{mg} \mathrm{ml}^{-1}$. The scale bar is $50 \mu \mathrm{m}$. (f) Photoluminescence spectra of the core (black line) and shell (red line) of the Ch-CNC droplets loaded with carbon dots, and an aqueous dispersion of carbon dots (blue line), both excited at $\lambda_{\text {exc }}=440 \mathrm{~nm}$. Inset: POM image of the droplet loaded with carbon dots. The scale bar is $50 \mu \mathrm{m}$. $\phi_{0}=0.043$ in $\mathbf{b}-\mathbf{f}$. 
a

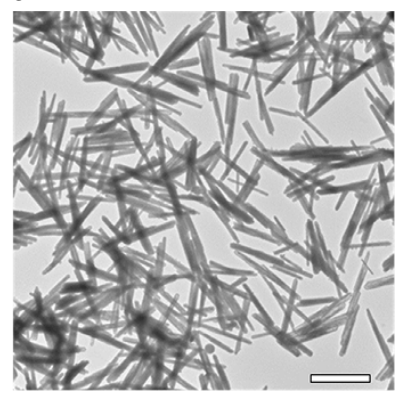

b

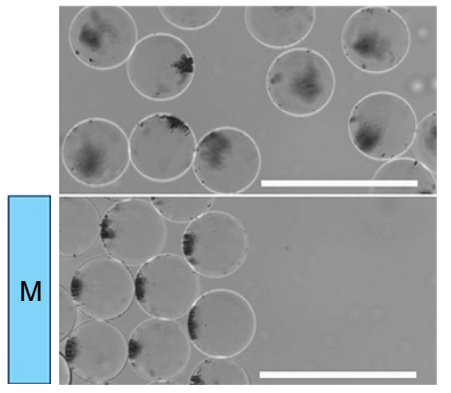

C

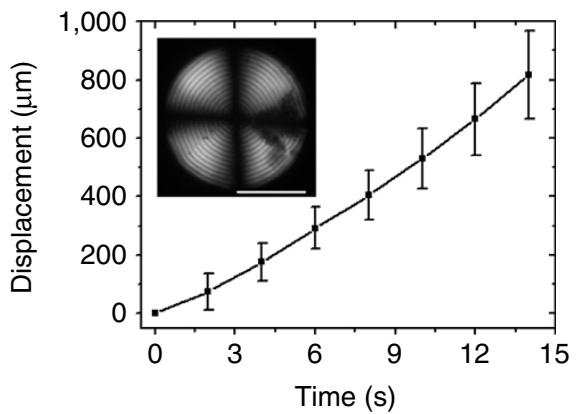

Figure 8 | Magnetic actuation of $\mathbf{C h}-\mathbf{C N C}$ droplets loaded with magnetic nanorods. (a) TEM images of magnetic $\mathrm{Fe}_{3} \mathrm{O}_{4} / \mathrm{SiO}_{2}$ rods. The $\mathrm{scale}$ bar is $1,000 \mathrm{~nm}$. (b) BF images of Ch-CNC droplets loaded with magnetic nanorods at concentration of $1.5 \mathrm{mg} \mathrm{ml}^{-1}$ without (top image) and with (bottom image) application of magnetic field. The scale bar is $250 \mu \mathrm{m}$. (c) Displacement of droplets loaded with $\mathrm{Fe}_{3} \mathrm{O}_{4} / \mathrm{SiO}_{2}$ rods under magnetic field, plotted as a function of time. Inset: POM image of corresponding droplet. The scale bar is $50 \mu \mathrm{m}$. The error bars represent the s.d. $\phi_{0}=0.043 \%$ in $\mathbf{b}$-c.

of droplets with $R=40 \mu \mathrm{m}$ ) the volume fraction of CNC in the shell, $\phi_{\mathrm{ch}}=0.0481$ would be only a little higher than the initial volume fraction of CNCs $\phi_{0}=0.048$. The difference $\phi_{\mathrm{ch}}-\phi_{0}$ becomes even smaller as the droplet radius increases. The small difference between $\phi_{c h}$ and $\phi_{0}$ explains why the Ch pitch measured in the shells of the biphasic Ch-CNC droplets is almost the same as in the macroscopic Ch phase.

The system of equations (1) and (2) can be solved only numerically ${ }^{44}$, however even a numerical treatment is problematic, since the thermodynamic characteristics of the system under study are not known. To obtain an order of magnitude estimate of the isotropic core radius, we approximate the free energy density difference in equation (1) through the chemical potentials of the isotropic $\left(\mu_{i}\right)$ and $\mathrm{Ch}\left(\mu_{\mathrm{ch}}\right)$ phases (Supplementary Note 2 ), as $f_{i}-f_{\mathrm{ch}} \approx\left(\mu_{i}-\mu_{\mathrm{ch}}\right) / v_{\mathrm{CNC}}$, where $v_{\mathrm{CNC}}$ is the volume of an individual CNC (Supplementary Methods). Furthermore, the difference between the chemical potentials can be estimated on the ground of translational-orientational entropy differences between the isotropic and $\mathrm{Ch}$ phases as $\mu_{i}-\mu_{\mathrm{ch}} \approx \alpha k_{B} T \phi_{0} b / v_{\mathrm{CNC}} \approx \alpha k_{B} T \phi_{0} L / D$. Here $\alpha$ is the numerical parameter of the order of 1 , which depends on the exact shape and size distribution of CNCs, $k_{B}$ is the Boltzmann constant, $T$ is the absolute temperature, and $b=\pi L^{2} D / 4$ is the average excluded volume of a CNC with a length $L$ and diameter $D$. In what follows, we denote $\left(\mu_{i}-\mu_{\mathrm{ch}}\right) / v_{\mathrm{CNC}} \approx 4 \alpha k_{B} T \phi_{0} /\left(\pi D^{3}\right)$ as $\Delta f$. To obtain an estimate for the equilibrium radius $r_{i}^{*}$ of the isotropic core, we assume that the interfacial tension $\sigma_{\text {chi }}$ and $\Delta f$ do not depend on the droplet size. By minimizing the energy in equation (1) with respect to $r_{i}$, one finds

$$
r_{i}^{*}=\frac{\sqrt{2 \Delta f\left(K_{1}-K_{24}\right)+\sigma_{\mathrm{chi}}^{2}}-\sigma_{\mathrm{chi}}}{\Delta f} .
$$

The interfacial tension $\sigma_{\text {chi }}$ between the isotropic and $\mathrm{Ch}$ phase formed by aqueous CNC suspensions is in the range of $10^{-7}-10^{-6} \mathrm{~J} \mathrm{~m}^{-2}$, as determined experimentally ${ }^{48}$. The value of $\Delta f$ defined above, for $\alpha=1$, is estimated to be $20 \mathrm{~J} \mathrm{~m}^{-3}$. For $K \sim 10 \mathrm{pN}$ (refs 38,39), as for other lyotropic liquid crystals, equation (3) predicts an equilibrium radius $r_{i}^{*}$ to be on the order of $1 \mu \mathrm{m}$, which is close to the experimentally measured core size. Note that for vanishingly small $\Delta f$, the equilibrium radius would be $r_{i}^{*}=\left(K_{1}-K_{24}\right) / \sigma_{\mathrm{chi}}$, thus potentially achieving the value in the range $10-100 \mu \mathrm{m}$.

This relatively large, $1 \mu \mathrm{m}$ or more, core radius is by two or even three orders of magnitude larger than a $10 \mathrm{~nm}$ core size of point defects in thermotropic liquid crystals ${ }^{44,45}$.The reason for the large core is that the quantity $\Delta f$ estimated above is significantly smaller than the corresponding latent heat of the isotropic-nematic transition in thermotropic liquid crystals. For typical thermotropic liquid crystals, for example, 5CB, the latent heat is on the order of $10^{6} \mathrm{~J} \mathrm{~m}^{-3}$ (refs 32,49), that is, five orders of magnitude higher than $\Delta f$ in the $\mathrm{Ch}-\mathrm{CNC}$ phase.

The reorganization from concentric to flattened packing of the Ch layers (Fig. 2) observed at decreasing $R / P$ ratio and caused by the finite surface anchoring at the droplet/F-oil interface was in qualitative agreement with earlier numerical simulations ${ }^{28}$ and experimental results ${ }^{37}$ for thermotropic $\mathrm{Ch}$ droplets. We ascribe this structural transition to the balance between the surface energy of the droplets (that scales as $W R^{2}$ ) and the elastic energy of $\mathrm{Ch}$ deformations (that scales linearly with the radius, as $K R)^{32}$. In large droplets (Fig. 2a), the surface anchoring of CNCs led to concentric packing of strongly curved layers, which remained parallel to the droplet surface, while in small droplets (Fig. 2c), the layers flattened at the expense of partial violation of boundary conditions. Tangential anchoring of the director at the $\mathrm{Ch} / \mathrm{F}-\mathrm{oil}$ interface acted to unwind the $\mathrm{Ch}$ helix, thus enlarging the $\mathrm{Ch}$ pitch, as observed experimentally.

The collective behaviour of colloid particles in the $\mathrm{Ch}-\mathrm{CNC}$ droplets was governed by the NP size. Earlier studies of phase separation in mixtures of rods and spheres ${ }^{16,50-53}$, as well as the discussion above on the appearance of the isotropic central core, suggest that phase separation of NPs in the Ch-CNC phase should be expected. The 54 and $184 \mathrm{~nm}$ latex NPs fit into the cores of defects and thus gained a freedom of translational motion within an isotropic phase. In the Ch shell, smaller $54 \mathrm{~nm}$ NPs were less disruptive to the $\mathrm{Ch}$ structure than larger $184 \mathrm{~nm}$ NPs, since the average distance between the CNCs is about $30 \mathrm{~nm}$ (ref. 29). Placement of $184 \mathrm{~nm}$ NPs into a Ch shell was apparently energetically more costly, as these NPs could distort the director. To calculate the actual energy cost of placing the NPs in the isotropic and Ch parts of the droplets more information is needed on surface interactions. The increase in the radius of the latex-rich isotropic cores with increasing $\phi_{\mathrm{NP}}$ stemmed from the stronger phase separation between the latexrich isotropic phase and the $\mathrm{CNC}$-rich $\mathrm{Ch}$ phase, as shown for the macroscopic mixture of the Ch-CNC phase and latex NPs (Supplementary Fig. 6c and Supplementary Note 1).

Larger $1,000 \mathrm{~nm}$-diameter latex beads and 1,000 $\mathrm{nm}$-long magnetic nanorods were observed in both the Ch shells and in the isotropic cores. The plausible reason is that these large particles are kinetically trapped in out-of-equilibrium states during the sample preparation, when the $\mathrm{Ch}-\mathrm{CNC}$ phase was mixed with the NPs. Large particles perturbed the Ch director around them and attracted each other through long-range elastic 
forces $^{50,54-56}$. If the surface anchoring at the NP-Ch interface is strong, the typical order of magnitude of the elastic energy of interaction is $\sim K d$, where $d$ is the size of the colloid particle. When $d$ is $1 \mu \mathrm{m}$, the elastic energy of interaction can reach $\sim 10^{-17} \mathrm{~J}$, that is, orders of magnitude higher than the thermal energy, $4 \times 10^{-21} \mathrm{~J}$. Therefore, large particles can form kinetically trapped clusters with irregular shapes that cannot relax into equilibrium shapes, due to very large (compared with thermal energy) energy barriers. A similar behaviour and physical mechanism have been described for the thermotropic $\mathrm{Ch}$ phase doped with $1 \mu \mathrm{m}$ silica particles, which formed irregular clusters and were not responsive to thermal annealing 57 .

Finally, our experiments show that the smallest $3.5 \mathrm{~nm}$ carbon dots and $10 \mathrm{~nm}$ gold NPs do not exhibit partitioning between different regions of the $\mathrm{Ch}$ droplets. These particles could fit between the CNCs and did not perturb the Ch structure.

In conclusion, progressive spherical confinement of $\mathrm{Ch}$ phases formed by rod-shape $\mathrm{CNC}$ colloids resulted in new structures and transitions between them. Notably, confinement-induced phase separation of the $\mathrm{Ch}-\mathrm{CNC}$ phase enabled a single-step preparation of core-shell droplets with a controllable Ch shell thickness, a method that is simpler than current methods for the preparation of liquid crystalline shells ${ }^{58,59}$. These structures can be used in fundamental studies of defects in frustrated liquid crystalline phases ${ }^{60}$, and for the engineering of particles with unusual mechanical and structural properties ${ }^{61,62}$. There is also a potential for optical applications of the droplets, since on evaporation of water from the $\mathrm{Ch}-\mathrm{CNC}$ phase, the pitch is reduced and the resulting material selectively reflects light in the visible spectral range $\mathrm{e}^{30}$. In aqueous suspensions, birefringence of CNCs normalized by their volume fraction is $\sim 0.12$ (ref. 27). For the volume fractions of CNCs used in our experiments, the effective birefringence (calculated for quasi-nematic regions of size significantly smaller than the pitch) is rather low, $\sim 0.006$, however, it can be increased by adding special ingredients such as gold nanorods ${ }^{6}$ or by increasing the volume fraction of $\mathrm{CNCs}^{27}$.

The co-assembly of CNCs with polymer and inorganic NPs provides a route for the design of new stimulus-responsive liquid crystalline materials. Previously, topological defects have been used as templates for the organization of colloid particles and molecules in thermotropic liquid crystals, which are mostly hydrophobic materials ${ }^{21,41,45,63-66}$. Our results demonstrate that the topological defect (droplet core) in a hydrophilic medium, such as an aqueous CNC suspension, can also be used for NP organization. The plasmonic, fluorescent and magnetic properties of the NP-loaded $\mathrm{Ch}-\mathrm{CNC}$ droplets broaden the range of potential applications which remain to be explored.

\section{Methods}

Generation and characterization of CNC droplets. The experimental details of the synthesis of NPs and the fabrication of microfluidic devices are given in the Supplementary Information (Supplementary Methods). The Ch-CNC droplets were produced by the emulsification of the aqueous $\mathrm{Ch}-\mathrm{CNC}$ phase in the flow-focusing microfluidic droplet generator ${ }^{31}$. The Ch phase (Supplementary Methods) was injected as a droplet phase at a flow rate of at $0.2 \mathrm{ml} \mathrm{h}^{-1}$ into the central channel of the microfluidic device using a syringe pump (PhD 200 Harvard Apparatus PHD 2000 Syringe Pump, USA). The continuous phase (fluorinated oil HFE-7500 or F-oil) mixed with 1 wt\% of the copolymer surfactant perfluoropolyether and poly(ethylene oxide-co-propylene oxide) was injected into the side channels of the microfluidic device using the second syringe pump (PhD 200 Harvard Apparatus PHD 2000 Syringe Pump, USA) at a flow rate varying in the range from 0.3 to $1.5 \mathrm{ml} \mathrm{h}^{-1}$. The $\mathrm{Ch}-\mathrm{CNC}$ droplets exiting the microfluidic device were collected in a $2 \mathrm{ml}$ glass vial and transferred into a cell consisting of two parallel glass slides separated by a $400 \mu \mathrm{m}$-thick spacer. The cell was sealed with epoxy glue and the droplets were equilibrated for various time intervals.
To prepare $\mathrm{Ch}-\mathrm{CNC}$ droplets with a broad size distribution and radii up to $115 \mu \mathrm{m}$, a mixture of $200 \mu \mathrm{l}$ of the Ch-CNC phase and $1 \mathrm{ml}$ of F-oil was shaken at $50 \mathrm{~Hz}$ for $1 \mathrm{~min}$ at room temperature in a vortex mixer. For the preparation of droplets with dimensions up to $250 \mu \mathrm{m}$, a mixture of $200 \mu \mathrm{l}$ of $\mathrm{Ch}-\mathrm{CNC}$ phase and $1 \mathrm{ml} \mathrm{F}$-oil containing $1 \mathrm{wt} \%$ of the copolymer surfactant was shaken at $30 \mathrm{~Hz}$ for $15 \mathrm{~s}$ at room temperature in a vortex mixer. The resultant emulsion of $\mathrm{Ch}-\mathrm{CNC}$ droplets was introduced in the cell, the cell was sealed with epoxy glue and the droplets were allowed to equilibrate for 7 days.

The BF and POM imaging, and the video recording of the $\mathrm{Ch}-\mathrm{CNC}$ droplets were carried out on an optical microscope (Olympus BX51) in the transmission mode. The diameters of the droplets and isotropic cores and the pitch of the $\mathrm{Ch}$ phase in the droplets were measured using the software ImageJ. The diameter of the isotropic droplet core was defined as the diameter of the innermost circle ring in the droplets, based on the BF images.

Generation and characterization of droplets loaded with NPs. The $\mathrm{Ch}-\mathrm{CNC}$ droplets loaded with NPs were prepared by emulsifying a mixture of the Ch-CNC phase and NPs in the flow-focusing microfluidic device. The procedure was identical to that used for the preparation of NP-free $\mathrm{Ch}-\mathrm{CNC}$ droplets. A mixture of $60 \mu \mathrm{l}$ of the suspension of FITC-labelled latex NPs, carbon dots, gold NPs or magnetic nanorods and $540 \mu \mathrm{l}$ of the $\mathrm{Ch}-\mathrm{CNC}$ phase was prepared by shaking at $50 \mathrm{~Hz}$ for $2 \mathrm{~min}$ at $25^{\circ} \mathrm{C}$ in a vortex mixer and immediately emulsifying this mixture in the microfluidic device.

The hybrid droplets were imaged using an optical microscope (Olympus BX51). The fluorescence images of the $\mathrm{Ch}$-CNC droplets loaded with FITC-labelled latex NPs and carbon dots were acquired on an inverted microscope (Nikon Eclipse-Ti). The fluorescence intensity profiles of these droplets were measured using a software (NIS-Elements AR Analysis). The Z-stack fluorescence images of the Ch-CNC droplets loaded with FITC-labelled latex NPs and carbon dots were taken by Nikon A1 confocal microscope. The extinction spectra of the solution of gold NPs were acquired using Cary-5000 spectrophotometer. The extinction spectra of the emulsion of the Ch-CNC droplets loaded with gold NPs were acquired using a plate reader (CLARIOstar, Mandel) by placing the emulsion of $\mathrm{Ch}-\mathrm{CNC}$ droplets in a 96-well plate. The photoluminescence of individual $\mathrm{Ch}-\mathrm{CNC}$ droplets loaded with carbon dots was measured by using a LabRam-HR (JY Horriba). The confocal spectrometer was attached to a microscope to focus on the targeted areas of the droplet. The photoluminescence was excited at $\lambda_{\text {exc }}=444 \mathrm{~nm}$. The videos of translational and rotational movement of the $\mathrm{Ch}-\mathrm{CNC}$ droplets loaded with magnetic nanorods were taken on an inverted microscope (Nikon Eclipse-Ti). Droplet displacement was measured by a software (NIS-Elements AR Analysis).

Data availability. The authors declare that the data supporting the findings of this study are available within the article and its Supplementary Information Files, and all relevant data are available from the authors.

\section{References}

1. Nie, Z., Petukhova, A. \& Kumacheva, E. Properties and emerging applications of self-assembled structures made from inorganic nanoparticles. Nat. Nanotech 5, 15-25 (2010).

2. Maye, M. M., Kumara, M. T., Nykypanchuk, D., Sherman, W. B. \& Gang, O. Switching binary states of nanoparticle superlattices and dimer clusters by DNA strands. Nat. Nanotech. 5, 116-120 (2010).

3. Velev, O. D. \& Gupta, S. Materials fabricated by micro- and nanoparticle assembly - the challenging path from science to engineering. Adv. Mater. 21, 1897-1905 (2009).

4. Wu, Y. et al. Composite mesostructures by nano-confinement. Nat. Mater. 3, 816-822 (2004).

5. Coursault, D. et al. Linear self-assembly of nanoparticles within liquid crystal defect arrays. Adv. Mater. 24, 1461-1465 (2012).

6. Liu, Q., Campbell, M. G., Evans, J. S. \& Smalyukh, I. I. Orientationally ordered colloidal co-dispersions of gold nanorods and cellulose nanocrystals. Adv. Mater. 26, 7178-7184 (2014).

7. Dammone, O. J. et al. Confinement induced splay-to-bend transition of colloidal rods. Phys. Rev. Lett. 109, 108303 (2012).

8. Soares e Silva, M. et al. Self-organized patterns of actin filaments in cell-sized confinement. Soft Matter 7, 10631-10641 (2011).

9. Teich, E. G., van Anders, G., Klotsa, D., Dshemuchadse, J. \& Glotzer, S. C. Clusters of polyhedra in spherical confinement. Proc. Natl Acad. Sci. USA 113 , E669-E678 (2016)

10. de Nijs, B. et al. Entropy-driven formation of large icosahedral colloidal clusters by spherical confinement. Nat. Mater. 14, 56-60 (2015).

11. Manoharan, V. N., Elsesser, M. T. \& Pine, D. J. Dense packing and symmetry in small clusters of microspheres. Science 301, 483-487 (2003).

12. Zhang, B. \& Cheng, X. Structures and dynamics of glass-forming colloidal liquids under spherical confinement. Phys. Rev. Lett. 116, 098302 (2016).

13. Shah, R. K., Kim, J. \& Weitz, D. A. Janus supraparticles by induced phase separation of nanoparticles in droplets. Adv. Mater. 21, 1949-1953 (2009). 
14. Wang, Y. et al. Colloids with valence and specific directional bonding. Nature 491, 51-55 (2012).

15. Tombolato, F., Ferrarini, A. \& Grelet, E. Chiral nematic phase of suspensions of rodlike viruses: left-handed phase helicity from a right-handed molecular helix. Phys. Rev. Lett. 96, 258302 (2006).

16. Adams, M., Dogic, Z., Keller, S. L. \& Fraden, S. Entropically driven microphase transitions in mixtures of colloidal rods and spheres. Nature 393, 349-352 (1998).

17. Song, W., Kinloch, I. A. \& Windle, A. H. Nematic liquid crystallinity of multiwall carbon nanotubes. Science 302, 1363 (2003).

18. Zamora-Ledezma, C. et al. Anisotropic thin films of single-wall carbon nanotubes from aligned lyotropic nematic suspensions. Nano Lett. 8, 4103-4107 (2008)

19. Wood, T. A., Lintuvuori, J. S., Schofield, A. B., Marenduzzo, D. \& Poon, W. C. K. A self-quenched defect glass in a colloid-nematic liquid crystal composite. Science 334, 79-83 (2011)

20. Poulin, P., Stark, H., Lubensky, T. C. \& Weitz, D. A. Novel colloidal interactions in anisotropic fluids. Science 275, 1770-1773 (1997)

21. Wang, X., Miller, D. S., Bukusoglu, E., De Pablo, J. J. \& Abbott, N. L. Topological defects in liquid crystals as templates for molecular self-assembly. Nat. Mater. 15, 106-112 (2016).

22. Milette, J. et al. Reversible long-range patterning of gold nanoparticles by smectic liquid. Soft Matter 8, 6593-6598 (2012).

23. Whitmer, J. K. et al. Nematic-field-driven positioning of particles in liquid crystal droplets. Phys. Rev. Lett. 111, 227801 (2013).

24. Lee, S. S. et al. Robust microfluidic encapsulation of cholesteric liquid crystals toward photonic ink capsules. Adv. Mater. 27, 627-633 (2015).

25. Humar, M., Ravnik, M., Pajk, S. \& Muševič, I. Electrically tunable liquid crystal optical microresonators. Nat. Photon. 3, 595-600 (2009).

26. Fan, J. et al. Light-directing omnidirectional circularly polarized reflection from liquid-crystal droplets. Angew. Chem. Int. Ed. 54, 2160-2164 (2015).

27. Frka-petesic, B., Sugiyama, J., Kimura, S., Chanzy, H. \& Maret, G. Negative diamagnetic anisotropy and birefringence of cellulose nanocrystals. Macromolecules 48, 8844-8857 (2015).

28. Seč, D., Porenta, T., Ravnik, M. \& Žumer, S. Geometrical frustration of chiral ordering in cholesteric droplets. Soft Matter 8, 11982-11988 (2012).

29. Schütz, C. et al. Rod packing in chiral nematic cellulose nanocrystal dispersions studied by small-angle X-ray scattering and laser diffraction. Langmuir 31, 6507-6513 (2015).

30. Lagerwall, J. P. F. et al. Cellulose nanocrystal-based materials: from liquid crystal self-assembly and glass formation to multifunctional thin films. NPG Asia Mater. 6, e80 (2014).

31. Xu, S. et al. Generation of monodisperse particles by using microfluidics: control over size, shape, and composition. Angew. Chem. Int. Ed. 44, 724-728 (2005).

32. Kleman, M. \& Lavrentovich, O. D. Soft Matter Physics: an introduction (Springer, 2003)

33. Dijkstra, M., Roij, V. R. \& Evans, R. Wetting and capillary nematization of a hard-rod fluid: a simulation study. Phys. Rev. E 63, 051703 (2001).

34. Aliabadi, R., Moradi, M. \& Varga, S. Orientational ordering of confined hard rods: the effect of shape anisotropy on surface ordering and capillary nematization. Phys. Rev. E 92, 032503 (2015).

35. Livolant, F. \& Leforestier, A. Condensed phases of DNA: structures and phase transitions. Prog. Polym. Sci. 21, 1115-1164 (1996).

36. Kurik, M. V. \& Lavrentovich, O. D. Topological defects of cholesteric liquid crystals for volumes with spherical shape. Mol. Cryst. Liq. Cryst. 72, 239-246 (1982).

37. Kurik, M. V. \& Lavrentovich, O. D. Negative-positive monopole transitions in cholesteric liquid crystals. J. Exp. Theor. Phys. Lett. 35, 444-447 (1982).

38. Zhou, S. et al. Elasticity of lyotropic chromonic liquid crystals probed by director reorientation in a magnetic field. Phys. Rev. Lett. 109, 037801 (2012).

39. Zhou, S., Cervenka, A. J. \& Lavrentovich, O. D. Ionic-content dependence of viscoelasticity of the lyotropic chromonic liquid crystal sunset yellow. Phys. Rev. E 90, 042505 (2014)

40. Voloschenko, D., Pishnyak, O. P., Shiyanovskii, S. V. \& Lavrentovich, O. D. Effect of director distortions on morphologies of phase separation in liquid crystals. Phys. Rev. E 65, 060701(R) (2002).

41. Pires, D., Fleury, J. \& Galerne, Y. Colloid particles in the interaction field of a disclination line in a nematic phase. Phys. Rev. Lett. 98, 247801 (2007).

42. Zhu, S. et al. Highly photoluminescent carbon dots for multicolor patterning, sensors, and bioimaging. Angew. Chem. Int. Ed. 52, 3953-3957 (2013).

43. Mottram, N. J. \& Sluckin, T. J. Defect-induced melting in nematic liquid crystals. Liq. Cryst. 27, 1301-1304 (2000).
44. Soulé, E. R. \& Rey, A. D. Hedgehog defects in mixtures of a nematic liquid crystal and a non-nematogenic component. Soft Matter 8, 1395-1403 (2012).

45. Wang, X. et al. Experimental insights into the nanostructure of the cores of topological defects in liquid crystals. Phys. Rev. Lett. 116, 147801 (2016).

46. Kim, Y., Shiyanovskii, S. V. \& Lavrentovich, O. D. Morphogenesis of defects and tactoids during isotropic - nematic phase transition in self-assembled lyotropic chromonic liquid crystals. J. Phys. Condens. Matter 25, 404202 (2013).

47. Lavrentovich, O. D., Ishikawa, T. \& Terentjev, E. M. Disclination loop in Mori-Nakanishi ansatz: role of the divergence elasticity. Mol. Cryst. Liq. Cryst. 299, 301-306 (1997)

48. Chen, W. \& Gray, D. G. Interfacial tension between isotropic and anisotropic phases of a suspension of rodlike particles. Langmuir 18, 633-637 (2002).

49. Gramsbergen, E. F., Longa, L. \& de Jeu, W. H. Landau theory of the nematic-isotropic phase transition. Phys. Rep. 135, 195-257 (1986).

50. Mondiot, F., Botet, R., Snabre, P., Mondain-monval, O. \& Loudet, J. Colloidal aggregation and dynamics in anisotropic fluids. Proc. Natl Acad. Sci. USA 111, 5831-5836 (2014).

51. Raghunathan, V. A., Richetti, P. \& Roux, D. Dispersion of latex particles in a nematic Solution. 2. phase diagram and elastic properties. Langmuir 12, 3789-3792 (1996).

52. Cuetos, A., Martínex-Haya, B., Lago, S. \& Rull, L. F. Use of Parsons-Lee and Onsager theories to predict nematic and demixing behavior in binary mixtures of hard rods and hard spheres. Phys. Rev. E 75, 061701 (2007).

53. Poulin, P., Francès, N. \& Mondain-monval, O. Suspension of spherical particles in nematic solutions of disks and rods. Phys. Rev. E 59, 4384-4387 (1999).

54. Muševič, I., Škarabot, M., Tkalec, U., Ravnik, M. \& Žumer, S. Two-dimensional nematic colloidal crystals self-assembled by topological defects. Science 313, 954-958 (2006).

55. Smalyukh, I. I., Lavrentovich, O. D., Kuzmin, A. N., Kachynski, A. V. \& Prasad, P. N. Elasticity-mediated self-organization and colloidal interactions of solid spheres with tangential anchoring in a nematic liquid crystal. Phys. Rev. Lett. 95, 157801 (2005).

56. Smalyukh, I. I., Kuzmin, A. N., Kachynski, A. V., Prasad, P. N. \& Lavrentovich, O. D. Optical trapping of colloidal particles and measurement of the defect line tension and colloidal forces in a thermotropic nematic liquid crystal. Appl. Phys. Lett. 86, 021913 (2005).

57. Zapotocky, M., Ramos, L., Poulin, P., Lubensky, T. C. \& Weitz, D. A. Particle-stabilized defect gel in cholesteric liquid crystals. Science 283, 209-212 (1999).

58. Utada, A. S. et al. Monodisperse double emulsions generated from a microcapillary device. Science 308, 537-541 (2005).

59. Fleischmann, E.-K. et al. One-piece micropumps from liquid crystalline core-shell particles. Nat. Commun. 3, 1178 (2012).

60. Lopez-Leon, T., Koning, V., Devaiah, K. B. S., Vitelli, V. \& Fernandez-Nieves, A. Frustrated nematic order in spherical geometries. Nat. Phys. 7, 391-394 (2011).

61. Donato, M. G. et al. Polarization-dependent optomechanics mediated by chiral microresonators. Nat. Commun. 5, 3656 (2014).

62. Tkachenko, G. \& Brasselet, E. Optofluidic sorting of material chirality by chiral light. Nat. Commun. 5, 3577 (2014).

63. Skarabot, M. et al. Hierarchical self-assembly of nematic colloidal superstructures. Phys. Rev. E 77, 061706 (2008).

64. Yoon, D. K. I. et al. Internal structure visualization and lithographic use of periodic toroidal holes in liquid crystals. Nat. Mater. 6, 866-870 (2007).

65. Kikuchi, H., Yokota, M., Hisakado, Y., Yang, H. \& Kajiyama, T. Polymerstabilized liquid crystal blue phases. Nat. Mater. 1, 64-68 (2002).

66. Blanc, C., Coursault, D. \& Lacaze, E. Ordering nano- and microparticles assemblies with liquid crystals. Liq. Cryst. Rev. 1, 83-109 (2013).

\section{Acknowledgements}

We thank Connaught Foundation and NSERC Canada (Discovery and Strategic grants) for financial support of this work. E.K. is grateful to the Canada Research Chair programs (NSERC CANADA). O.L. acknowledges financial support from NSF DMR-1121288 and NSF DMS-1434185. B.Y. acknowledges financial support from The National Science Foundation of China (NSFC) under Grant No. 51373065. Y.L. acknowledges Banting Postdoctoral Fellowships program, administered by the Government of Canada and the International Postdoctoral Exchange Fellowship Program 2013 funded by the Office of China Postdoctoral Council (20130035). We are grateful to Professor Michael Rubinstein (The University of North Carolina) and Dr Oleg Gang (Brookhaven National Laboratory) for fruitful discussions. The authors thank Xiguang Gao for initiating preliminary experiments and Ilya Gourevich for assistance in imaging experiments. 


\section{Author contributions}

E.K. and Y.L. conceived the concept of this work and designed the experiments. Y.L. conducted the experiments and analysed data. J.J.-y.S. and E.P. prepared microfluidic devices and conducted microfluidic emulsification. E.M.L acquired images of nanoparticles. A.K. synthesized gold nanoparticles. H.T.-A. synthesized latex particles. S.Z. and B.Y. synthesized the carbon dots. O.L. and E.K. contributed in the interpretation and discussion of the results. A.H. conducted optical characterization of hybrid droplets. E.K., Y.L. and O.L. wrote the paper.

\section{Additional information}

Supplementary Information accompanies this paper at http://www.nature.com/ naturecommunications

Competing financial interests: The authors declare no competing financial interests.
Reprints and permission information is available online at http://npg.nature.com/ reprintsandpermissions/

How to cite this article: Li, Y. et al. Colloidal cholesteric liquid crystal in spherical confinement. Nat. Commun. 7:12520 doi: 10.1038/ncomms12520 (2016).

(c) (i) This work is licensed under a Creative Commons Attribution 4.0 International License. The images or other third party material in this article are included in the article's Creative Commons license, unless indicated otherwise in the credit line; if the material is not included under the Creative Commons license, users will need to obtain permission from the license holder to reproduce the material. To view a copy of this license, visit http://creativecommons.org/licenses/by/4.0/

(C) The Author(s) 2016 